\title{
Clinical and radiographic predictors of acute compartment syndrome in the treatment of tibial shaft fractures: a retrospective cohort study
}

Lydia Wuarin, Amanda I. Gonzalez, Matthieu Zingg, Patrick Belinga, Pierre Hoffmeyer, Robin Peter, Anne Lübbeke and Axel Gamulin *(i)

\begin{abstract}
Background: The purpose of this study was to evaluate the association between epidemiological, clinical and radiographic factors of patients with tibial shaft fractures and the occurrence of acute compartment syndrome.

Methods: 270 consecutive adult patients sustaining 273 tibial shaft fractures between January 2005 and December 2009 were included in this retrospective cohort study. The outcome measure was acute compartment syndrome. Patient-related (age, sex), fracture-related (high- vs. low-energy injury, isolated trauma vs. polytrauma, closed vs. open fracture) and radiological parameters (AO/OTA classification, presence or absence of a noncontiguous tibial plateau or pilon fracture, distance from the centre of the tibial fracture to the talar dome, distance between tibial and fibular fracture if associated, and angulation, translation and over-riding of main tibial fragments) were evaluated regarding their potential association with acute compartment syndrome. Univariate analysis was performed and each covariate was adjusted for age and sex. Finally, a multivariable logistic regression model was built, and odds ratios and 95\% confidence intervals were obtained. Statistical significance was defined as $p<0.05$.

Results: Acute compartment syndrome developed in 31 (11.4\%) cases. In the multivariable regression model, four covariates remained statistically significantly associated with acute compartment syndrome: polytrauma, closed fracture, associated tibial plateau or pilon fracture and distance from the centre of the tibial fracture to the talar dome $\geq 15 \mathrm{~cm}$.

Conclusions: One radiological parameter related to the occurrence of acute compartment syndrome has been highlighted in this study, namely a longer distance from the centre of the tibial fracture to the talar dome, meaning a more proximal fracture. This observation may be useful when clinical findings are difficult to assess (doubtful clinical signs, obtunded, sedated or intubated patients). However, larger studies are mandatory to confirm and refine the prediction of acute compartment syndrome occurrence. Radiographic signs of significant displacement were not found to be correlated to acute compartment syndrome development. Finally, the higher rate of acute compartment syndrome occurring in tibial shaft fractures associated to other musculoskeletal, thoraco-abdominal or cranio-cerebral injuries must raise the level of suspicion of any surgeon managing multiply injured patients.
\end{abstract}

Keywords: Tibial shaft fracture, Tibial diaphysis fracture, Acute compartment syndrome, Fasciotomy, Risk factors

* Correspondence: axel.gamulin@hcuge.ch

Division of Orthopaedic and Trauma Surgery, University Hospitals of Geneva,

4 Rue Gabrielle-Perret-Gentil, CH-1211 Geneva, Switzerland

(c) The Author(s). 2020 Open Access This article is distributed under the terms of the Creative Commons Attribution 4.0 International License (http://creativecommons.org/licenses/by/4.0/), which permits unrestricted use, distribution, and reproduction in any medium, provided you give appropriate credit to the original author(s) and the source, provide a link to the Creative Commons license, and indicate if changes were made. The Creative Commons Public Domain Dedication waiver (http://creativecommons.org/publicdomain/zero/1.0/) applies to the data made available in this article, unless otherwise stated. 


\section{Background}

Tibial shaft fractures represent approximately $2 \%$ of all fractures [1]. The AO/OTA classification might be used for their description [2]. Tibial shaft fracture patterns range from low-energy undisplaced fractures to high-energy multifragmented or segmental displaced fractures [3]. The energy delivered to the limb during trauma may also cause soft-tissue lesions, including skin lacerations and acute compartment syndrome (ACS) [4]. Occurrence of ACS in tibial shaft fractures is reported to reach $11.5 \%$ [5-8].

At time of assessment by the orthopedic surgeon, patients may be intubated and/or sedated and/or obtunded, and clinical evaluation of ACS (pain out of proportion with the clinical findings and exacerbated by passive muscle stretch) may be impossible to perform [9-13]. In addition to intra-compartmental pressure (ICP) measurement, alternative ACS predictors need to be identified [11, 14, 15].

Research on risk factors for the occurrence of ACS after tibial shaft fractures identified some demographic, injury-related, and clinical predictors, such as young age, male sex, mechanism of injury, high-energy trauma and open fracture $[4-8,16-18]$. Three recent studies on potential radiographic predictors of ACS after tibial shaft fractures failed to identify any of them, including $\mathrm{AO} /$ OTA classification, fracture length ratio, associated fibular fracture and its distance to the tibial shaft fracture, or segmental tibial shaft fracture $[5,7,16]$.

However, some potential radiographic indicators of high-energy trauma in tibial shaft fractures (which might be likely to cause ACS more frequently) have not been studied yet. These potential risk factors include: 1) the displacement (angulation, translation and over-riding) of the tibial shaft fracture (it was hypothesized that higher displacement was associated with higher-energy trauma); 2) the location of the fracture within the tibial diaphysis (it was hypothesized that a trauma producing a more proximal fracture was delivering more energy within a bulkier muscle environment); and 3) the presence of an associated non-contiguous plateau or pilon fracture (it was hypothesized that a second fracture complex separated from the tibial shaft fracture and located either in the proximal or distal metaphysis was a sign of a higher-energy trauma causing two distinct fracture patterns) [19].

To the authors' knowledge, these potential risk factors were not simultaneously evaluated to date. The objective of this study was therefore to analyze the relation between key demographic, injury-related, clinical and radiographic factors in patients with tibial shaft fractures and the subsequent development of ACS.

\section{Methods}

\section{Ethics statement}

Before starting the study, approval was delivered by the institutional research ethics committee.

\section{Study population and design}

The setting of this retrospective cohort study was a 1900-bed urban academic medical centre delivering primary to tertiary care to 500,000 inhabitants.

The institutional hospitalization diagnoses database was used to identify all consecutive patients admitted with a diaphyseal tibia fracture. Inclusion criteria were: 1) a diaphyseal tibia fracture as defined by the AO/OTA classification code 42 [2], with or without metaphyseal involvement or extension to the knee or ankle joint, and with or without associated fibular fracture; 2) trauma as the cause of fracture; 3) hospital admission between January 2005 and December 2009; 4) definitive treatment in the authors' institution; and 5) age $>16$ years old. At this point, 297 patients with 301 fractures were identified. Exclusion criteria were: 1) hospital admission more than $24 \mathrm{~h}$ after initial trauma (no occurrence); 2) pathological fracture (no occurrence); 3) periprosthetic or peri-implant fracture (five fractures); 4) open growth plates (one fracture); 5) transfer to another institution for definitive treatment (one fracture); 6) above or below knee amputation within the first $24 \mathrm{~h}$ after the trauma (no occurrence); 7) death within the first $24 \mathrm{~h}$ after the trauma (no occurrence); and 8) radiographic images incomplete for full evaluation and measurements (e.g. only one incidence available; 21 fractures).

Finally, 270 patients with 273 fractures were included in the analysis (Fig. 1). The 21 cases with incomplete radiological data were comparable to the 273 studied cases regarding sex, mean age and incidence of ACS (Table 1).

\section{Outcome}

The outcome was occurrence of ACS leading to fasciotomy. Using the same method as in a previously published study, ICP measurements were not routinely performed to diagnose ACS [19]. When standard clinical evaluation was equivocal (patients with unclear clinical signs and those intubated, sedated or obtunded), ICP was measured in each of the four leg compartments using an Intra-Compartmental Pressure Monitor (Stryker Osteosynthesis AG, Biberist, Switzerland) or an arterial line transducer system with a beveled 18-gauge needle [20]. When ICP was not measured, ACS was confirmed only if muscle bulging or suffering was reported in the operative notes. If muscle was not described in the operative notes, ACS was confirmed only if ICP measurements were pathological.

\section{Variables of interest}

Patients' charts were reviewed to extract information on age, sex, injury mechanism, isolated trauma or polytrauma, closed or open fracture and Gustilo classification $[21,22]$ in case of open fractures. In order to distinguish low- and high-energy trauma, injury mechanism was further classified as either "fall from own height" or "other" 


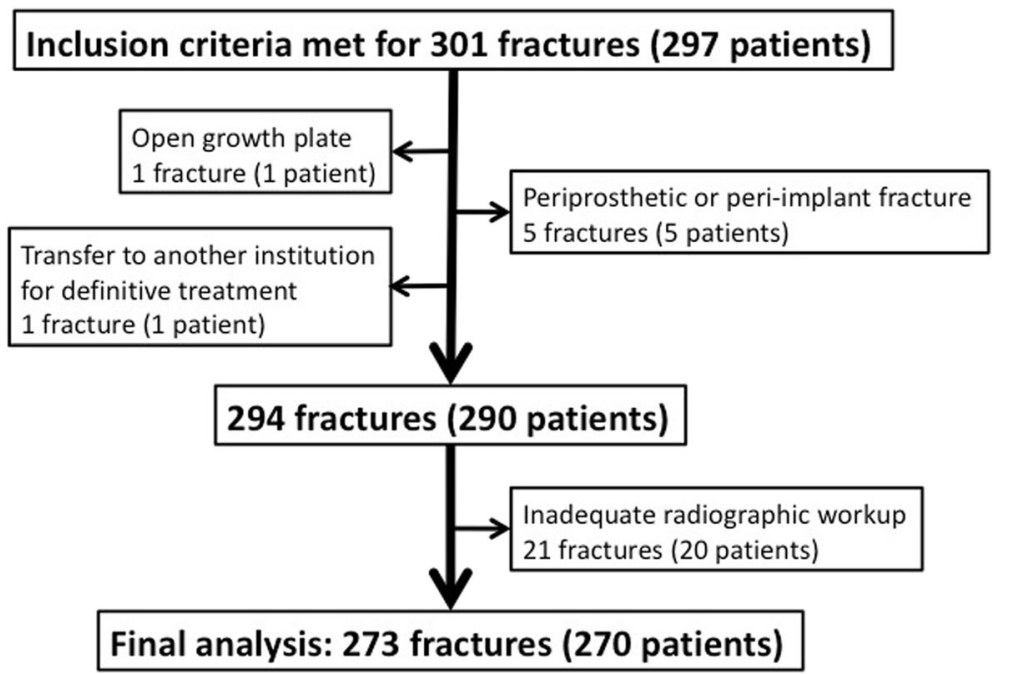

Fig. 1 Flowchart depicting patients and fractures meeting inclusion and exclusion criteria, with final numbers available for analysis

(falls from more than one meter high, sports or fight injuries, traffic accidents, crushes, construction site accidents). For statistical analysis, fractures were considered in two ways (closed or open) and in three categories (closed, Gustilo type 1, and Gustilo type 2 and 3).

Radiographic evaluation of all cases was performed using a dedicated descriptive form including detailed illustrations of classification schemes and radiographic measurements. Based on the initial set of radiographs, AO/OTA classifications were determined, as well as the presence or absence of a non-contiguous tibial plateau or pilon fracture. Only the first level of the AO/OTA classification (42-A, B and C) was used for the statistical analysis, as inter-observer variability concerning the sub-classification 1,2 and 3 remains a concern [23]. Non-contiguous tibial plateau or pilon fractures were defined as a second fracture complex separated from the tibial shaft fracture and located either in the proximal (AO/OTA 41 classification) or distal (AO/OTA 43 classification) metaphysis [2]. Radiographic measurements were performed on the initial antero-posterior and lateral plain radiographs of the leg after gross realignment of the limb (as per institutional policy) using a dedicated webbased open-source PACS workstation DICOM viewer (Weasis medical viewer, available on https://nroduit.github. io/en/). A descriptive chart was used to perform the analysis in a reproducible way (Figs. 2, 3 and 4). The following measures were obtained: 1 ) distance from talar dome surface to middle of tibial fracture in $\mathrm{mm}$ (Fig. 2; the proximal and distal ends of the tibial fracture were determined taking into account potential intermediate fragments, and the midpoint of the line linking these both points was defined as the center of the tibial fracture); 2) distance from talar dome surface to middle of fibular fracture in $\mathrm{mm}$ (Fig. 2; the proximal and distal ends of the fibular fracture were determined taking into account potential intermediate fragments, and the midpoint of the line linking these both points was defined as the center of the fibular fracture); 3) distance from tibial fracture to fibular fracture in $\mathrm{mm}$, which was measured and also calculated by substracting value of point 1 from value of point 2 (Fig. 2): the mean value between measured distance and calculated distance was used for the analysis; 4 ) over-riding of tibial fracture in

Table 1 Incidence of ACS, sex, and age compared between the population of 270 patients with 273 fractures included in the final analysis and the population of 20 patients with 21 fractures meeting exclusion criteria due to incomplete available radiographic workup

\begin{tabular}{llll}
\hline & $\begin{array}{l}\text { Final analysis } \\
273 \text { fractures (270 patients) }\end{array}$ & $\begin{array}{l}\text { Patients excluded due to } \\
\text { incomplete radiographic workup } \\
21 \text { fractures (20 patients) }\end{array}$ \\
\hline Male sex, $n$ (\%) & $201(73.6 \%)$ & $14(66.7 \%)$ & $P .456^{\text {a }}$ \\
Age, mean \pm SD & $39.9 \pm 15.4$ & $42.6 \pm 16.6$ & $0.441^{\mathrm{b}}$ \\
ACS occurrence, $n(\%)$ & $31(11.4 \%)$ & $2(9.5 \%)$ & $1.000^{\mathrm{a}}$ \\
\hline
\end{tabular}

ACS acute compartment syndrome

${ }^{\text {a }}$ Fisher's exact test

b T-test 


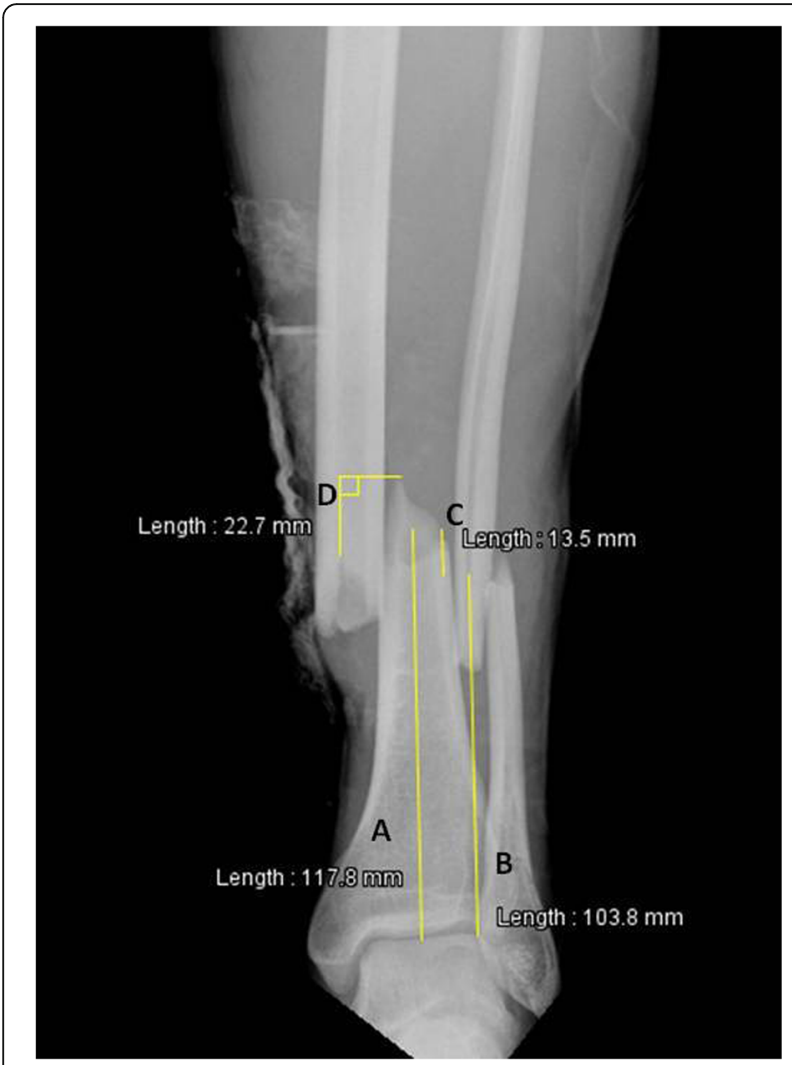

Fig. 2 Plain antero-posterior radiograph of a left distal leg including the ankle, with an AO/OTA 42 A2.3 fracture, in a 33.5 years old male patient. Line A represents the distance from talar dome surface to middle of tibial fracture $(118 \mathrm{~mm}$ in this case); the proximal and distal ends of the tibial fracture were determined taking into account potential intermediate fragments, and the midpoint of the line linking these both points was defined as the center of the tibial fracture (this step is not represented on the figure). Line B represents the distance from talar dome surface to middle of fibular fracture (104 $\mathrm{mm}$ in this case); the proximal and distal ends of the fibular fracture were determined taking into account potential intermediate fragments, and the midpoint of the line linking these both points was defined as the center of the fibular fracture (this step is not represented on the figure). In this example, a third spike fragment located at the posterior border of the proximal main fragment and visible on the lateral radiograph (Fig. 4) was present, and its position was anticipated when drawing the proximal and distal end points of the fracture on the antero-posterior radiograph; for this reason, the center of the fibular fracture seems close to what appears to be the distal end of the fracture on the antero-posterior radiograph. Line $C$ represents the distance from tibial fracture to fibular fracture $(14 \mathrm{~mm}$ in this case). Line $D$ represents over-riding of tibial fracture, which is measured between two corresponding bony landmarks on both main fragments ( $23 \mathrm{~mm}$ in this case)

$\mathrm{mm}$ (Fig. 2); 5) translation at the level of the fracture center as a ratio relative to the tibial diameter on the anteroposterior incidence (Fig. 3); 6) translation at the level of the fracture center as a ratio relative to the tibial diameter on the lateral incidence (Fig. 4); 7) total translation ratio which was calculated with the formula.

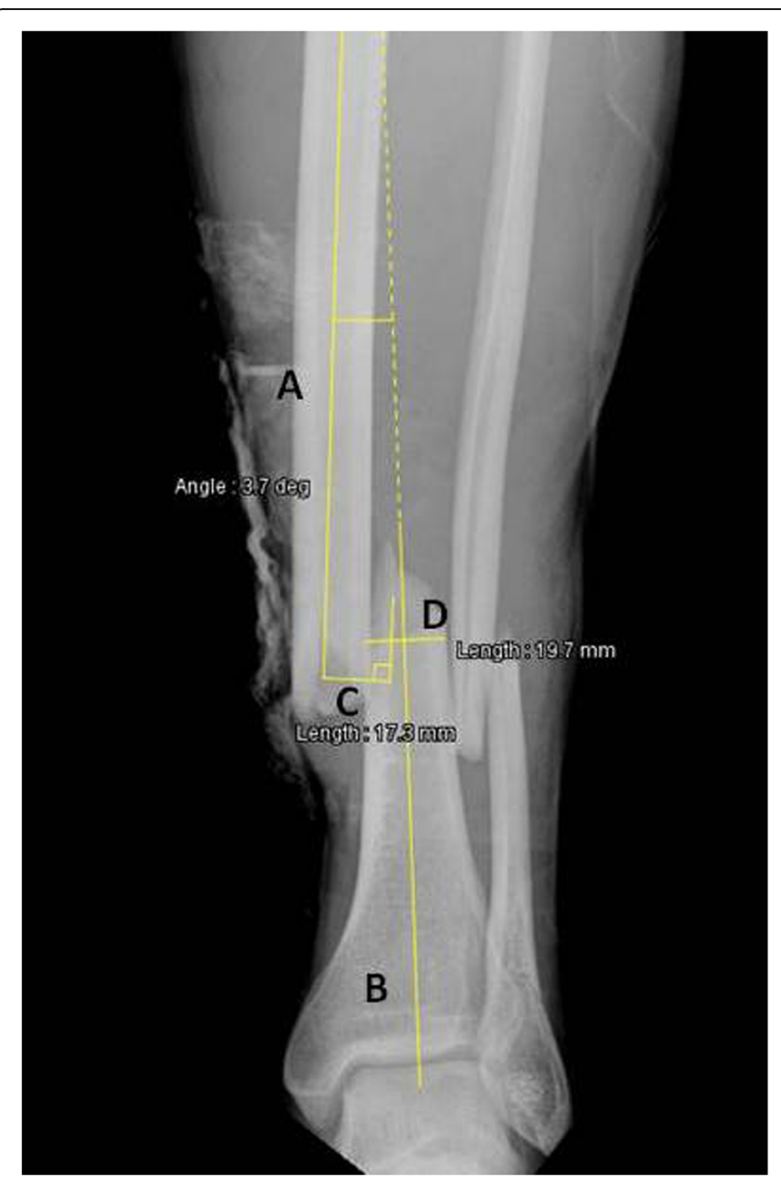

Fig. 3 Plain antero-posterior radiograph of a left distal leg including the ankle, with an AO/OTA 42 A2.3 fracture, in a 33.5 years old male patient. Line A represents the anatomical axis of the proximal tibial fragment (the line was drawn in the center of the diaphysis), and line B the anatomical axis of the distal tibial fragment (the line was drawn from the middle of the tibial plafond/talar dome to the center of the diaphysis). Line $C$ represents translation at the level of the fracture center, relative to the axis of the proximal fragment. Line $D$ represents the diameter of the tibia at the level of the fracture. Translation is expressed as a ratio relative to the tibial diameter using the formula Line C/Line D; in this case, 17.3/19.7= 0.88 . Angulation is measured between Lines $A$ and $B$; in this case, $3.7^{\circ}$

$$
\sqrt{(\text { value of point } 5)^{2}+(\text { value of point } 6)^{2}}
$$

8) angulation on antero-posterior incidence (Fig. 3; the angle was measured between the anatomical axis of the distal fragment, and the anatomical axis of the proximal fragment); 9) angulation on lateral incidence (Fig. 4; the angle was measured between lines tangential to the inner or outer border of the anterior cortex of each main fragment, depending on which one of these both borders was the more recognizable on radiographs); 10) and total angulation, which was calculated with the formula. 


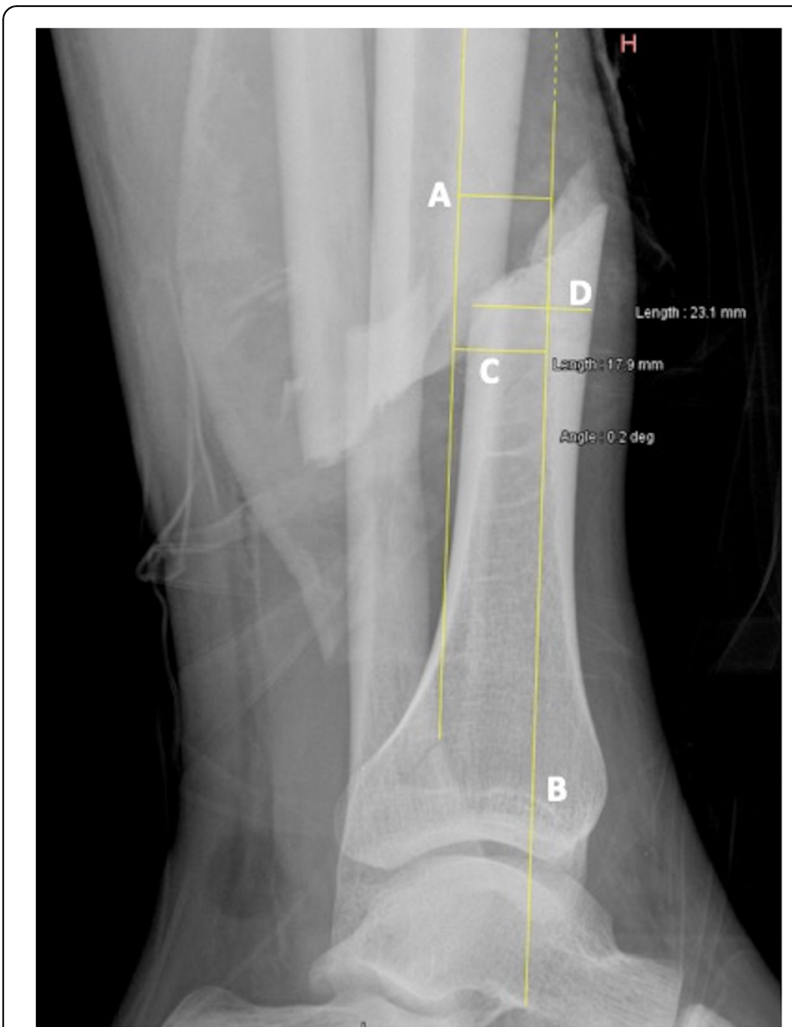

Fig. 4 Plain lateral radiograph of a left distal leg including the ankle, with an AO/OTA 42 A2.3 fracture, in a 33.5 years old male patient. Line A represents the axis of the proximal tibial fragment, and line $B$ the axis of the distal tibial fragment; these lines are tangential to the inner or outer border of the anterior cortex, depending on which one of these both borders is the more recognizable on radiograph. Line $C$ represents translation at the level of the fracture center, relative to the axis of the proximal fragment. Line D represents the diameter of the tibia at the level of the fracture. Translation is expressed as a ratio relative to the tibial diameter using the formula Line C/Line $D$; in this case, 17.9/23.1 $=0.77$. Angulation is measured between Lines $\mathrm{A}$ and $\mathrm{B}$; in this case, $0.2^{\circ}$

$\tan ^{-1} \sqrt{\tan ^{2}(\text { value of point } 8)+\tan ^{2}(\text { value of point } 9)}$.

The choice of talar dome instead of tibial plafond to measure the distance to the tibial and fibular fractures was driven by three reasons: a common reference independent from both bones involved by the fracture (tibia and fibula) was preferred; some fractures of the tibial shaft can extend into the tibial plafond and potentially distort its radiological anatomy thus making the choice of a measurement reference on its surface difficult; as initial radiographs were assessed, measurements could be performed before any realignment or external fixation potentially provoking joint space distraction and artificial distance increase.

Radiographic analysis of all cases was first performed by a single surgeon, who was blinded to the outcome (LW, 2 years of experience in orthopedic radiographic interpretation and measurements like the above-described ones). In order to address the possible issue of inter-observer reliability, an Internet based random integer generator (www.random.org) was used to randomly select 30 cases among the 273 fractures. As cases were picked independently from each other, duplicates may occur that were excluded from the reliability assessment. A second orthopedic surgeon, which was trauma-fellowship trained, performed complete radiographic analysis on the randomly picked radiographs (AG, more than 15 years of experience in orthopedic radiographic interpretation and measurements like the above-described ones).

In the cases of ACS occurrence, the delay between injury and ACS and between surgery and ACS (before, during or after surgery), the timing of fasciotomy (during first intervention or later), and the compartment pressure measurements as well as the description of the muscle compartments during fasciotomy (bulging, suffering, necrosis) were extracted from patients' charts.

\section{Statistical analysis}

Clinical characteristics of patients and fractures with and without ACS were compared using frequency distributions for categorical variables and means and standard deviations for continuous variables. Fischer's exact test was used for categorical variables and t-test for continuous variables. To evaluate the association of the covariates with ACS, we used logistic regression analysis to obtain univariate odds ratios (OR) and 95\% confidence intervals (95\% CI). For this analysis, approximate midpoints were used to dichotomize continuous variables (age $<45$ years, distance talar domemiddle of tibial fracture $\geq 15 \mathrm{~cm}$, distance fibular fracturetibial fracture $<3 \mathrm{~cm}$, total angulation $\geq 5^{\circ}$, total translation $\geq 40 \%$, and over-riding $\geq 8 \mathrm{~mm}$ ).

Each covariate was then separately adjusted for age and sex using multivariable logistic regression analysis, because the risk of ACS may be related to the energy dissipated at the time of injury and younger men may be more prone to sustain high-energy trauma.

Finally, variables with significance $<0.1$ were included in a final multivariable logistic regression model, and ORs and $95 \%$ CIs were obtained. Statistical significance was defined as $p<0.05$ in this final model.

Intraclass correlation coefficients (ICC two-way-random) were obtained to quantify the inter-observer reliability of the radiographic analysis. The ICC is a special case of the weighted kappa and has been considered equivalent [24]. Results were interpreted following the Landis and Koch recommendations: $\kappa<0=$ poor agreement, $\kappa$ 0.0 $-0.20=$ slight agreement, $\kappa \quad 0.21-0.40=$ fair agreement, $\kappa \quad 0.41-0.60=$ moderate agreement, к $0.61-0.80=$ substantial /good agreement, $\kappa$ 0.81-1.00 = almost perfect agreement [25].

IBM $^{\oplus}$ SPSS $^{\oplus}$ Statistics version 22.0 and 25.0 software (IBM SPSS, Chicago, IL) was used for statistical analysis. 


\section{Results}

All 273 fractures in 270 patients had complete epidemiological and clinical data as well as complete radiographic measurements available for analysis. Overall, ACS developed in 31 (11.4\%) cases. Of these 31 cases, 23 had ICP measurement confirmation and eight had operative notes describing either muscle bulging or suffering. There were no operative notes describing muscle necrosis. There was no false positive diagnosis of ACS occurrence, as every patient who underwent fasciotomy had either pathological ICP measurements before fasciotomy or muscle bulging or suffering described at the time of fasciotomy.

Nine patients were diagnosed with ACS preoperatively. Of those, five were treated with intramedullary nailing (IMN), three with external fixation, and one with plate osteosynthesis; in all cases, surgery occurred within $24 \mathrm{~h}$ after admission (range one to $19 \mathrm{~h}$ ), and the first procedure was four compartment fasciotomy, followed by skeletal fixation. Peroperative ACS (ACS diagnosed after completion of osteosynthesis and treated within the same surgical time) occurred in 12 patients (11 IMN and one external fixator); in all cases, surgery was performed within $28 \mathrm{~h}$ after admission (range two to $28 \mathrm{~h}$ ). Finally, 10 ACS were diagnosed after the patient had left the operative theatre following fracture fixation (nine IMN and one external fixation performed between two and $24 \mathrm{~h}$ after admission); patients were brought back to the operative theater for four compartment fasciotomy between seven hours and 9 days after initial fixation.

Table 2 shows demographics, injury characteristics and clinical and radiographic factors of the study patients.

In the univariate analysis (Table 3 ), age $<45$ years, male gender, high-energy trauma, polytrauma, closed fracture, associated tibial plateau or pilon fracture and longer distance from the centre of the tibial fracture to the talar dome $(\geq 15 \mathrm{~cm}$, meaning a more proximal fracture) were associated with an increased rate of ACS with a significance level set at $p<0.1$. After separate adjustment of each variable for age and sex, there were only small changes in significance levels, except for high-energy trauma. The latter association was attenuated after adjustment and no longer statistically significant.

In the final multivariable regression model (Table 4), four covariates remained statistically significantly associated with ACS: polytrauma, closed fracture, associated tibial plateau or pilon fracture and distance from the centre of the tibial fracture to the talar dome $\geq 15 \mathrm{~cm}$.

Inter-observer reliability showed almost perfect agreement for complete and first level AO/OTA classification, distance talar dome - middle of tibial fracture, total angulation and total translation, and substantial or good agreement for over-riding (Table 5). Of note, two duplicates were excluded from the 30 randomly picked cases, leaving 28 cases $(10.3 \%$ of 273 cases) for the final analysis.

Table 2 Demographics, injury characteristics, clinical and radiographic factors of the 270 study patients with 273 tibial shaft fractures

\begin{tabular}{|c|c|c|}
\hline & $\begin{array}{l}\text { ACS absent } \\
N=242(88.6 \%)\end{array}$ & $\begin{array}{l}\text { ACS present } \\
N=31(11.4 \%)\end{array}$ \\
\hline Age (years), mean $\pm S D$ & $40.6 \pm 15.9$ & $34.4 \pm 9.9$ \\
\hline Male sex, $n(\%)$ & $174(71.9 \%)$ & $27(87.1 \%)$ \\
\hline High energy trauma, $n(\%)$ & $168(69.4 \%)$ & $28(90.3 \%)$ \\
\hline Polytrauma, $n$ (\%) & $52(21.5 \%)$ & $13(41.9 \%)$ \\
\hline Soft tissue closed, $n$ (\%) & $158(65.3 \%)$ & $25(80.6 \%)$ \\
\hline Gustilo I, $n(\%)$ & $42(17.4 \%)$ & $2(6.5 \%)$ \\
\hline Gustilo II-III, n (\%) & $42(17.4 \%)$ & $4(12.9 \%)$ \\
\hline AO/OTA classification A, $n$ (\%) & $145(59.9 \%)$ & $14(45.2 \%)$ \\
\hline$B, n(\%)$ & $77(31.8 \%)$ & $12(38.7 \%)$ \\
\hline C, $n(\%)$ & $20(8.3 \%)$ & $5(16.1 \%)$ \\
\hline Associated non-contiguous tibial plateau or pilon fracture, $n(\%)$ & $3(1.2 \%)$ & $2(6.5 \%)$ \\
\hline Distance talar dome-middle of tibial fracture $(\mathrm{mm})$, mean $\pm \mathrm{SD}$ & $135.3 \pm 59.1$ & $176.8 \pm 68.7$ \\
\hline Total angulation $\left({ }^{\circ}\right)$, mean \pm SD & $6.6 \pm 5.0$ & $7.0 \pm 5.0$ \\
\hline Total translation (\%), mean \pm SD & $46.1 \pm 27.3$ & $42.2 \pm 29.1$ \\
\hline Over-riding $(\mathrm{mm})$, mean $\pm \mathrm{SD}$ & $9.3 \pm 7.9$ & $9.1 \pm 7.7$ \\
\hline Associated fibular fracture, $n(\%)^{a}$ & $200(82.6 \%)$ & $26(83.9 \%)$ \\
\hline Distance fibular fracture-tibial fracture $(\mathrm{mm})$, mean $\pm \mathrm{SD}^{\mathrm{a}}$ & $99.6 \pm 81.4$ & $88.3 \pm 74.4$ \\
\hline
\end{tabular}

ACS acute compartment syndrome

a 200 of the 242 cases without ACS had an associated fibula fracture, as well as 26 of the 31 cases with ACS. Altogether, there were 47 cases without associated fibula fracture 
Table 3 Association between variables of interest (demographics, injury characteristics and clinical and radiographic factors) and the occurrence of acute compartment syndrome: univariate analysis and after adjustment for age and sex

\begin{tabular}{|c|c|c|c|c|}
\hline & Univariate analysi & & Each variable adju & and sex \\
\hline & OR $(95 \% \mathrm{Cl})$ & $P$ value & OR $(95 \% \mathrm{Cl})$ & $P$ value \\
\hline Age $<45$ years. & $3.30(1.23 ; 8.90)$ & 0.018 & - & - \\
\hline Male sex & $2.64(0.89 ; 7.82)$ & 0.080 & - & - \\
\hline High energy trauma & $4.11(1.21 ; 13.95)$ & 0.023 & $2.76(0.78 ; 9.77)$ & 0.117 \\
\hline Polytrauma & $2.64(1.21 ; 5.74)$ & 0.014 & $2.29(1.03 ; 5.05)$ & 0.041 \\
\hline Closed fracture & $2.22(0.87 ; 5.61)$ & 0.094 & $2.57(0.99 ; 6.64)$ & 0.051 \\
\hline AO/OTA types B and C & $1.82(0.86 ; 3.85)$ & 0.121 & $1.63(0.75 ; 3.51)$ & 0.215 \\
\hline Associated tibial plateau or pilon fracture & $5.49(0.88 ; 34.26)$ & 0.068 & $5.24(0.77 ; 35.46)$ & 0.090 \\
\hline Distance talar dome-middle of tibial fracture $\geq 15 \mathrm{~cm}$ & $4.40(2.02 ; 9.58)$ & $<0.001$ & $3.54(1.59 ; 7.87)$ & 0.002 \\
\hline Total angulation $\geq 5^{\circ}$ & $1.29(0.60 ; 2.77)$ & 0.520 & $1.15(0.53 ; 2.53)$ & 0.725 \\
\hline Total translation $\geq 40 \%$ & $0.77(0.36 ; 1.63)$ & 0.493 & $0.87(0.40 ; 1.89)$ & 0.721 \\
\hline Over-riding $\geq 8 \mathrm{~mm}$ & $0.97(0.46 ; 2.04)$ & 0.928 & $1.03(0.48 ; 2.22)$ & 0.946 \\
\hline Associated fibular fracture & $1.09(0.39 ; 3.01)$ & 0.865 & $1.49(0.53 ; 4.23)$ & 0.446 \\
\hline Distance fibular fracture-tibial fracture $<3 \mathrm{~cm}$ & $0.97(0.39 ; 2.45)$ & 0.951 & $1.20(0.46 ; 3.08)$ & 0.712 \\
\hline
\end{tabular}

OR odds ratio, 95\% Cl 95\% confidence interval

\section{Discussion}

This study highlighted an $11.4 \%$ risk of ACS occurrence during the treatment of tibial shaft fractures. Higher distance from the talar dome to the centre of the tibial fracture, associated tibial plateau or pilon fracture, closed fracture and polytrauma were individually confirmed by the multivariable regression analysis as significantly associated with ACS.

Reported incidence rates of ACS in the treatment of tibial shaft fractures range from 3 to $11.5 \%$ and seem to be stable over the past decades $[5-8,16]$. Our results fall within this range. In this aspect, our study population represents a rather usual cohort of tibial shaft fracture patients. We did not find any clinical records suspect of late ACS sequellae in the charts of patients that were not diagnosed with ACS, and every patient who underwent fasciotomy had pathological ICP values before fasciotomy, and/or presented muscle bulging or suffering at the time of fasciotomy.
Some ACS developed during or after surgery (external or definitive internal fixation) and the surgical procedure may therefore be partly responsible for these occurrences. However, we believe that surgery only represents an aggravating factor, and that the initial fracture with soft tissue injury is the primary factor for the occurrence of ACS. Indeed, after the initial injury, there may be a certain period of soft tissue vulnerability to further surgical aggression, as recently postulated [19].

In the univariate analysis, patients younger than 45 years were about three times as likely to present with ACS than patients over 45 years. However, the multivariable analysis did not confirm this association as statistically significant. Young age is the most consistently observed independent predictor of ACS occurrence throughout the literature $[5,7,8,18,26]$. The reasons for ACS occurring more often in younger individuals are thought to be that younger patients tend to have bulkier muscle and thicker, less yielding fasciae [5, 18]. Thus,

Table 4 Association between variables of interest (demographics, injury characteristics and clinical and radiographic factors) and the occurrence of acute compartment syndrome: multivariable analysis

\begin{tabular}{lll}
\hline & Multivariate analysis & $P$ value \\
\cline { 2 - 3 } & OR $(95 \% \mathrm{Cl})$ & 0.132 \\
\hline Age $<45$ years. & $2.26(0.78 ; 6.55)$ & 0.112 \\
Male sex & $2.61(0.80 ; 8.52)$ & 0.045 \\
Polytrauma & $2.58(1.02 ; 6.49)$ & 0.004 \\
Closed fracture & $5.47(1.73 ; 17.32)$ & 0.047 \\
Associated tibial plateau or pilon fracture & $8.80(1.03 ; 75.02)$ & 0.005 \\
Distance talar dome-middle of tibial fracture $\geq 15 \mathrm{~cm}$ & $3.49(1.47 ; 8.27)$ & \\
\hline
\end{tabular}

OR odds ratio, 95\% Cl 95\% confidence interval 
Table 5 Inter-observer reliability assessment of radiographic analysis

\begin{tabular}{ll}
\hline & Intraclass correlation coefficient (95\% Cl) \\
\hline AO/OTA classification, complete & $0.876(0.750-0.941)$ \\
AO/OTA classification, first level (42-A, B and C) & $0.927(0.849-0.966)$ \\
Distance talar dome-middle of tibial fracture & $0.982(0.961-0.991)$ \\
Total angulation & $0.944(0.882-0.974)$ \\
Total translation & $0.917(0.827-0.961)$ \\
Over-riding & $0.734(0.490-0.871)$ \\
\hline
\end{tabular}

Intraclass correlation coefficient; values < 0 represent poor agreement, values between 0.0 and 0.20 slight agreement, values between 0.21 and 0.40 fair agreement, values between 0.41 and 0.60 moderate agreement, values between 0.61 and 0.80 substantial or good agreement, and values between 0.81 and 1.00 almost perfect agreement. 95\% Cl 95\% confidence interval

any increase in intra-compartmental volume is more likely to lead to a rapid rise in ICP and to ACS.

The univariate analysis showed a trend for male patients to be more likely to develop ACS, but statistical significance was not reached in the multivariable analysis. Literature on this subject is contradictory, with some reports showing male sex to be a risk factor for ACS [18], and some others failing to highlight an association between gender and ACS occurrence [5, 7, 8].

This study failed to point out an association between high-energy trauma and development of ACS, although there was a statistically significant association in the univariate analysis. The literature is controversial on this topic, as one study showed an association between highenergy trauma and ACS occurrence [6] while others did not $[5,7,18]$. Indeed, retrospective determination of the amount of energy released during initial trauma might be unreliable, as chart review may not provide a good representation of the detailed mechanism of injury (for example, type of sports injury, vehicle speed, etc.) [19]. In this perspective, our retrospective attempt to differentiate between low and high-energy mechanism by classifying trauma into "fall from own height" and "other" makes any conclusion about energy of the injury weak.

Interestingly, differentiation between isolated trauma and polytrauma seems to be better in determining possible association with ACS development, as polytrauma was found to be associated with ACS occurrence in the multivariable analysis. This differentiation did not use the Injury Severity Score (ISS) [27, 28], but rather relied on a subjective appreciation of the extension of trauma: the fractured leg (tibia and/or fibula) was either an isolated injury or associated to other musculoskeletal, thoraco-abdominal, spine or cranio-cerebral injuries. This subjective appreciation can be quickly performed by the treating surgeon during the initial survey of any trauma patient and is probably more reliable than any anamnestic investigation in determining the potential risk of ACS occurrence. Furthermore, it does not rely on an a posteriori ISS determination, which is usually performed several hours after the trauma [27, 28]. However, to mitigate this statement, it is important to note that despite an increase in high-energy trauma as a causative injury and an improved survival rate among severe polytrauma patients noted over the past decades [29, 30], ACS rates following tibial shaft fractures do not seem to have increased during the same period $[5-8,16]$.

Despite older reports recognizing open fractures as positively associated with the occurrence of ACS in tibial shaft fractures, with an incidence of ACS directly proportional to the severity of the open fracture [4, 17], our results are more in line with recent publications having not found any association between open fractures and ACS development $[5,7,8]$. Our results even show that open fractures might have a protective effect against ACS development. Importantly, these findings must not lead clinicians to be wrongly reassured by an open fracture, assuming that the wound would relieve the pressure inside the muscle compartments, as ACS may still develop in these occurrences [5-8]. In fact, skin wounds in the vicinity of a fracture should be recognized as a direct sign of increased amount of underlying fascial and muscle injury and must not be underestimated by the treating physician [19].

Although the first level of the AO/OTA classification (42-A, B and C) is supposed to reflect the amount of energy delivered to the bone and surrounding soft tissues to produce the fracture [23], this parameter did not reach statistical significance in the multivariable analysis. There was nevertheless a distinctive trend for higher-grade fractures to develop ACS, with 14 of 159 42-A fractures (8.8\%), 12 of 89 42-B (13.5\%) and 5 of 25 42-C (20\%). Another study found similar results several years ago [7]. Thus, this radiographic parameter should be used with caution in evaluating the potential risk for ACS to occur. The incidence of ACS in the setting of 42-A fractures should not be underestimated or discarded, and the index of suspicion should remain high.

The presence of an associated non-contiguous tibial plateau or pilon fracture was highlighted in this study. It represents a red flag and an indicator of a high amount of energy transmitted to the injured limb, causing 
increased skeletal lesions (associated non-contiguous tibial plateau or pilon fracture) and extensive soft tissue damage potentially leading to the development of ACS. Similar results were recently published, where the presence of a non-contiguous tibial fracture or knee dislocation was a predictor of ACS development after tibial plateau fractures [19]. However, this finding is statistically weak, due to low numbers of events (three in the group without ACS and two in the group with ACS) leading to extended CIs.

The most powerful factor highlighted by this study in predicting occurrence of ACS during the treatment of tibial shaft fractures is the distance between the talar dome and the centre of the tibial fracture. In other words, the more proximal the tibial fracture is, the more likely ACS will occur. A previous study found that the incidence of ACS was higher with tibial plateau fractures than with tibial shaft or pilon fractures, but there were no specific results presented on fracture localization within the tibial shaft [16]. This factor has been specifically evaluated only once in the literature, and no influence of the localization of the fracture within the tibial shaft (proximal vs. middle vs. distal third) on the development of ACS could be demonstrated [6]. Our finding is new and may be explained by the fact that a fracture occurring at a location surrounded by a bulkier muscle mass (proximal diaphysis) may lead to more energy transmitted to the soft tissues, thus to the potential development of ACS. This observation may be useful when clinical findings are difficult to assess (doubtful clinical signs, obtunded, sedated or intubated patients). However, the design of the present study did not allow calculating this distance as a ratio relative to the total length of the tibia, which could have been an interesting element to generalize the use of this finding. This could be the aim of a further study on this topic.

One of our initial hypotheses, postulating that higher fracture displacement would be associated to higherenergy trauma and more extended soft tissue damages leading to ACS, could not be confirmed by this study. No association between angulation, translation or overriding at the fracture site and occurrence of ACS could be demonstrated. As per institutional policy, grossly deformed limbs are aligned by gentle traction and rotational control before any radiographs are taken, in order to protect vascularization and to relieve soft tissue suffering of the injured limb as soon as possible. Thus, radiographs may not reflect the initial deformity and this makes any conclusion about fracture displacement weak.

Additionally, neither the presence or absence of an associated fibular fracture nor the distance between the tibial fracture and the fibular fracture when present showed an association with ACS development. This is in line with previously published data [16].
Despite being one of the largest series to date analyzing the association between key demographic, injuryrelated, clinical and radiographic parameters in tibial shaft fracture patients and the development of ACS, this study suffers several limitations: 1) the retrospective attempt to differentiate between low and high-energy trauma on chart review makes any conclusions about mechanism or energy of the injury weak; 2) ICP measurements, which would have been the gold standard to diagnose or exclude ACS, were performed only on a subset of patients, thus introducing the possibility of false positive or false negative diagnosis; however, we did not find any clinical records suspect of late ACS sequellae in the charts of patients that were not diagnosed with ACS, and every patient who underwent fasciotomy had pathological ICP values before fasciotomy, and/or presented muscle bulging or suffering at the time of fasciotomy; 3) radiographic analysis was performed on one occasion only thus not quantifying intra-observer variability and measurement imprecision; however, interobserver reliability assessment performed on $10.3 \%$ of the cases showed almost perfect agreement for all measurements, except for over-riding which had substantial or good agreement: this may validate the entire radiographic measurement process performed by an observer with 2 years of experience in orthopedic radiographic interpretation and measurements, as the observer who took part in the inter-observer reliability assessment had more than 15 years of experience in the field; 4) the most powerful factor highlighted by this study in predicting occurrence of ACS during the treatment of tibial shaft fractures, namely the distance between the talar dome and the centre of the tibial fracture, was measured as a distance rather than as a ratio relative to the total length of the tibia; the design of the present study did not allow such a measurement, and this ratio determination could be the aim of a further study in order to generalize the use of this finding; 5) and finally, proximal tibiofibular dislocation, which has been recently shown to be possibly associated with a higher rate of ACS (29\%) in both tibial plateau and tibial shaft fractures, was not investigated in the present study [31].

\section{Conclusions}

One radiological parameter related to the occurrence of ACS has been highlighted in this study, namely a longer distance from the centre of the tibial fracture to the talar dome, meaning a more proximal fracture within the tibial shaft. A potential explanation is that a fracture occurring at a location surrounded by a bulkier muscle mass (proximal diaphysis) may lead to more energy transmitted to the soft tissues, thus to the potential development of ACS. This observation may be useful when clinical findings are difficult to assess (doubtful clinical signs, obtunded, sedated or intubated patients). However, larger studies are 
mandatory to confirm and refine the prediction of ACS occurrence, and to develop this finding into an easily usable ratio relative to the total length of the tibia. Radiographic signs of major displacement were not found to be correlated to ACS occurrence and can therefore not be used in the assessment of ACS risk in case of tibial shaft fracture. Finally, the higher rate of ACS occurring in tibial shaft fractures when associated to other musculoskeletal, thoracoabdominal, spine or cranio-cerebral injuries must raise the level of suspicion of any surgeon managing multiply injured patients.

\section{Abbreviations}

95\% Cl: 95\% confidence intervals; ACS: Acute compartment syndrome; AO/ OTA: Arbeitsgemeinschaft für Osteosynthesefragen / Orthopaedic Trauma Association; DICOM: Digital imaging and communications in medicine; ICP: Intra-compartmental pressure; IMN: Intramedullary nailing; ISS: Injury severity score; OR: Odds ratios; PACS: Picture archiving and communication system

\section{Acknowledgements}

Not applicable.

\section{Authors' contributions}

LW carried out the conception and design of the study, was the major contributor for the acquisition and interpretation of data and drafted and critically revised the manuscript. AIG participated in the conception and design of the study, performed the statistical analysis, contributed to the interpretation of data and drafted and critically revised the manuscript. MZ developed and validated the trigonometric formula used for calculating total angulation and critically revised the manuscript. PB contributed to the acquisition and interpretation of data and critically revised the manuscript. $\mathrm{PH}$ participated in the conception and design of the study and critically revised the manuscript. RP participated in the conception and design of the study and critically revised the manuscript. AL participated in the statistical analysis and critically revised the manuscript. AG carried out the conception and design of the study, contributed to the acquisition and interpretation of data and drafted and critically revised the manuscript. Finally, all authors gave their final approval of the version to be published. They all agree to be accountable for all aspect of the work in ensuring that questions related to the accuracy or integrity of any part of the work are appropriately investigated and resolved.

\section{Funding}

There was no external source of funding or financial support for this study.

\section{Availability of data and materials}

The datasets used and/or analyzed during the current study are available from the corresponding author on reasonable request.

\section{Ethics approval and consent to participate}

Before initiation of the study, approval was obtained from the institutional research ethics board (Commission Centrale d'Ethique de la Recherche sur I'Etre Humain des Hôpitaux Universitaires de Genève, reference number 10190 / NAC 10-063). All procedures performed in studies involving human participants were in accordance with the ethical standards of the institutional and/or national research committee and with the 1964 Helsinki declaration and its later amendments or comparable ethical standards. For this type of study formal consent to participate is not required.

\section{Consent for publication}

Not applicable.

\section{Competing interests}

The authors declare that they have no competing interests.
Received: 30 May 2019 Accepted: 6 January 2020

Published online: 13 January 2020

\section{References}

1. Court-Brown CM, Caesar B. Epidemiology of adult fractures: a review. Injury. 2006;37:691-7.

2. Kellam JF, Meinberg EG, Agel J, Karam MD, Roberts CS. Fracture and dislocation classification Compendium-2018. J Orthop Trauma. 2018; 32(Suppl 1):1-170.

3. Trafton PG. Tibial shaft fractures. In: Browner BD, Jupiter JB, Levine AM, Trafton PG, Krettek C, editors. Skeletal trauma: basic science, management, and reconstruction. 3rd ed. Philadelphia: Saunders Elsevier; 2003. p. 2131255.

4. Blick SS, Brumback RJ, Poka A, Burgess AR, Ebraheim NA. Compartment syndrome in open tibial fracture. J Bone Joint Surg Am. 1986;68-A:1348-53.

5. McQueen MM, Duckworth AD, Aitken SA, Sharma RA, Court-Brown CM. Predictors of compartment syndrome after tibial fracture. J Orthop Trauma. 2015;29:451-5.

6. Menetrey J, Peter R. Acute compartment syndrome in the post-traumatic leg. Rev Chir Ortho Reparatrice Appar Mot. 1998;84:272-80.

7. Park S, Ahn J, Gee AO, Kuntz AF, Esterhai JL. Compartment syndrome in tibial fractures. J Orthop Trauma. 2009;23:514-8.

8. Shadgan B, Pereira G, Menon M, Jafari S, Darlene Reid W, O'Brien PJ. Risk factors for acute compartment syndrome of the leg associated with tibial diaphyseal fractures in adults. J Orthop Traumatol. 2015;16:185-92.

9. Amendola A, Twaddle BC. Compartment syndromes. In: Browner BD, Jupiter JB, Levine AM, Trafton PG, Krettek C, editors. Skeletal trauma: basic science, management, and reconstruction. 3rd ed. Philadelphia: Saunders Elsevier; 2003. p. 268-92

10. Gorczyca JT, Roberts CS, Pugh KJ, Ring D. Review of treatment and diagnosis of acute compartment syndrome of the calf: current evidence and best practices. Instr Course Lect. 2011;60:35-42.

11. Mauser N, Gissel H, Henderson C, Hao J, Hak D, Mauffrey C. Acute lower-leg compartment syndrome. Orthopedics. 2013;36:619-24.

12. Olson SA, Glasgow RR. Acute compartment syndrome in lower extremity musculoskeletal trauma. J Am Acad Orthop Surg. 2015;13:436-44.

13. Südkamp NP. Soft-tissue injury: pathophysiology and its influence on fracture management - evaluation/classification of closed and open injuries. In: Rüedi TP, Murphy WM, editors. AO principles of fracture management. 2nd ed. Stuttgart: Thieme; 2007. p. 86-113.

14. Nelson JA. Compartment pressure measurements have poor specificity for compartment syndrome in the traumatized limb. J Emerg Med. 2013;44: 1039-44.

15. Nudel I, Dorfmann L, de Botton G. The compartment syndrome: is the intracompartment pressure a reliable indicator for early diagnosis? Math Med Biol. 2017;34:547-58.

16. Allmon C, Greenwell P, Paryavi E, Dubina A, O'Toole RV. Radiographic predictors of compartment syndrome occurring after tibial fracture. J Orthop Trauma. 2016;30:387-91.

17. DeLee JC, Stiehl JB. Open tibia fracture with compartment syndrome. Clinical Orthop Relat Res. 1981;160:175-84.

18. McQueen MM, Gaston P, Court-Brown CM. Acute compartment syndrome. Who is at risk? J Bone Joint Surg Br. 2000;82:200-3.

19. Gamulin A, Lübbeke A, Belinga P, Hoffmeyer P, Perneger TV, Zingg M, Cunningham G. Clinical and radiographic predictors of acute compartment syndrome in the treatment of tibial plateau fractures: a retrospective cohort study. BMC Musculoskelet Disord. 2017;18:307.

20. Wilson SC, Vrahas MS, Berson L, Paul EM. A simple method to measure compartment pressures using an intravenous catheter. Orthopedics. 1997; 20:403-6.

21. Gustilo RB, Anderson JT. Prevention of infection in the treatment of one thousand and twenty-five open fractures of long bones: retrospective and prospective analyses. J Bone Joint Surg Am. 1976;58-A:453-8.

22. Gustilo RB, Mendoza RM, Williams DN. Problems in the management of type III (severe) open fractures: a new classification of type III open fractures. J Trauma. 1984;24:742-6.

23. Marsh JL, Slongo TF, Agel J, Broderick JS, Creevey W, DeCoster TA, Prokuski L, Sirkin MS, Ziran B, Henley B, Audigé L. Fracture and dislocation classification compendium - 2007: Orthopaedic trauma association classification, database and outcomes committee. J Orthop Trauma. 2007; 21(Suppl):S1-133. 
24. Fleiss $\mathrm{JL}$, Cohen $\mathrm{J}$. The equivalence of weighted kappa and the intraclass correlation coefficient as measures of reliability. Educ Psychol Meas. 1976;33: 613-9.

25. Landis JR, Koch GG. The measurement of observer agreement for categorical data. Biometrics. 1977;33:159-74.

26. Elliott KG, Johnstone AJ. Diagnosing acute compartment syndrome. J Bone Joint Surg (Br). 2003;85:625-32.

27. Baker SP, O'Neill B, Haddon W, Long WB. The injury severity score: a method for describing patients with multiple injuries and evaluating emergency care. J Trauma. 1974;14:187-96.

28. Copes WS, Champion HR, Sacco WJ, Lawnick MM, Keast SL, Bain LW. The injury severity score revisited. J Trauma. 1988;28:69-77.

29. Röding F, Lindkvist M, Bergström U, Lysholm J. Epidemiologic patterns of injuries treated at the emergency department of a Swedish medical center. Injury Epidemiol. 2015;2:3.

30. Trauma Register DGU •. 20 Years of trauma documentation in GermanyActual trends and developments. Injury. 2014;45(Suppl 3):S14-9.

31. Herzog GA, Serrano-Riera R, Sagi HC. Traumatic proximal tibiofibular dislocation: a marker of severely traumatized extremities. J Orthop Trauma. 2015:29:456-9.

\section{Publisher's Note}

Springer Nature remains neutral with regard to jurisdictional claims in published maps and institutional affiliations.

Ready to submit your research? Choose BMC and benefit from:

- fast, convenient online submission

- thorough peer review by experienced researchers in your field

- rapid publication on acceptance

- support for research data, including large and complex data types

- gold Open Access which fosters wider collaboration and increased citations

- maximum visibility for your research: over $100 \mathrm{M}$ website views per year

At BMC, research is always in progress.

Learn more biomedcentral.com/submissions 